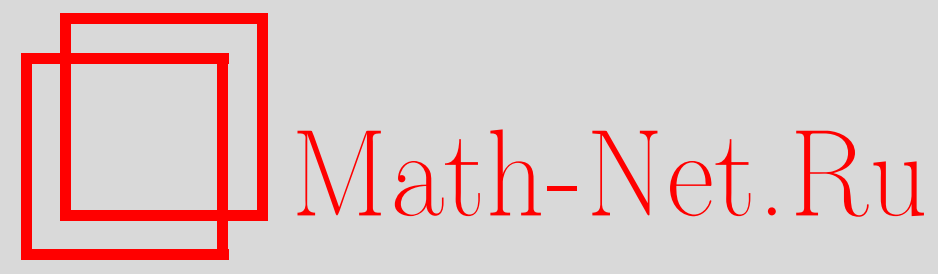

С. С. Акбаров, Строение модулей над стереотипной алгеброй операторов $\mathcal{L}(X)$, Функи. анализ и его прил., 2006, том 40, выпуск 2, 1-12

DOI: https://doi.org/10.4213/faa2

Использование Общероссийского математического портала Math-Net.Ru подразумевает, что вы прочитали и согласны с пользовательским соглашением http://www. mathnet.ru/rus/agreement

Параметры загрузки:

IP : 34.227 .88 .159

26 апреля 2023 г., 14:23:01

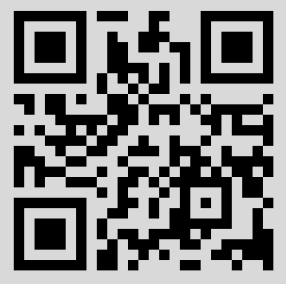


Функииональный анализ и его приложения

2006, т. 40, вып. 2, с. 1-12

УДК 517.982.1+517.986.2

\title{
Строение модулей над стереотипной алгеброй операторов $\mathscr{L}(\boldsymbol{X})$
}

\author{
(c) 2006. С. C. Акьаров*
}

\section{§1. Постановка задачи и главные результаты}

Один из классических результатов теории ассоциативных алгебр (см., например, [1]) гласит, что всякий модуль $M$ над алгеброй $\mathscr{L}(X)$ операторов на конечномерном векторном пространстве $X$ (например, над $\mathbb{C}$ ) представим в виде прямой суммы $\bigoplus_{\mathfrak{m}} X(\mathfrak{m}$ - кардинальное число, зависящее от $M)$ некоторого семейства $\left\{X_{i} ; i \in \mathfrak{m}\right\}$ экземпляров пространства $X$ :

$$
M=\bigoplus_{\mathfrak{m}} X
$$

Этот факт не переносится на случай бесконечномерного пространства $X$, потому что в противном случае сама алгебра $\mathscr{L}(X)$, рассматриваемая как левый модуль над собой, имела бы такой же вид, $\mathscr{L}(X)=\bigoplus_{\mathfrak{m}} X$, откуда следовало бы, что $\mathscr{L}(X)$ состоит только из конечномерных операторов.

Действительно, предположим, что такой изоморфизм существует и обозначим его через $\iota: \bigoplus_{\mathfrak{m}} X \rightarrow \mathscr{L}(X)$. Пусть $\left\{x_{i} ; i \in \mathfrak{m}\right\} \in \bigoplus_{\mathfrak{m}} X-$ семейство, в котором члены со всеми индексами, кроме фиксированного индекса $i_{0}$, равны нулю,

$$
x_{i}=\left\{\begin{array}{ll}
a, & i=i_{0} \\
0, & i \neq i_{0} .
\end{array} \quad(a \in X),\right.
$$

Тогда одномерные операторы вида $g \otimes a$, где $g(a)=1$, должны действовать на образ $\iota\left(\left\{x_{i}\right\}\right)$ этого семейства тождественно:

$$
\begin{aligned}
(g \otimes a) \cdot \iota\left(\left\{x_{i}\right\}\right) & =\iota\left((g \otimes a) \cdot\left\{x_{i}\right\}\right)=\iota\left(\left\{\begin{array}{ll}
(g \otimes a) \cdot a, & i=i_{0} \\
(g \otimes a) \cdot 0, & i \neq i_{0}
\end{array}\right\}\right) \\
& =\iota\left(\left\{\begin{array}{ll}
a, & i=i_{0} \\
0, & i \neq i_{0}
\end{array}\right\}\right)=\iota\left(\left\{x_{i}\right\}\right),
\end{aligned}
$$

и это означает, что $\iota\left(\left\{x_{i}\right\}\right)$ - одномерный оператор в $\mathscr{L}(X)$. Поскольку любое семейство $\left\{x_{i} ; i \in \mathfrak{m}\right\} \in \bigoplus_{\mathfrak{m}} X$ является конечной суммой семейств, в которых члены со всеми индексами, кроме одного, равны нулю, мы получаем, что образ $\iota\left(\left\{x_{i}\right\}\right)$ произвольного семейства $\left\{x_{i} ; i \in \mathfrak{m}\right\} \in \bigoplus_{\mathfrak{m}} X$ должен быть конечномерным оператором, т. е. любой оператор в $\mathscr{L}(X)$ конечномерен. Разумеется, это невозможно при бесконечномерном $X$, и мы получаем противоречие.

Если прямую сумму $\bigoplus_{\mathfrak{m}} X$ представлять себе как тензорное произведение пространства $X$ на какое-то другое векторное пространство $E$ (алгебраической

*Работа поддержана грантом РФФИ №05-01-00982. 
размерности $\mathfrak{m})$, то сказанное можно будет сформулировать в виде следующего предложения.

ПРЕДЛОЖЕНИЕ. Если $X$ - конечномерное векторное пространство над $\mathbb{C}$, то для всякого левого модуля $M$ над алгеброй $\mathscr{L}(X)$ линейных операторов на $X$ существует единственное с точностью до изоморфизма векторное пространство $E$ (над тем жее полем $\mathbb{C}$ ), такое, что справедлив изоморбизм модулей

$$
M \cong E \otimes X
$$

где умножение в $E \otimes X$ задается формулой

$$
\varphi \cdot(e \otimes x)=e \otimes \varphi(x), \quad x \in X, e \in E, \varphi \in \mathscr{L}(X) .
$$

Если же $X$ бесконечномерно, то не всякий $\mathscr{L}(X)$-модуль представим в ви$\partial e(1)$.

Итак, имеется качественная разница между конечномерным и бесконечномерным пространствами $X$, и, зная это, естественно поинтересоваться, справедливо ли представление, подобное (1), в ситуации, когда $X$ наделено какойнибудь дополнительной структурой, например, топологией? (При этом, разумеется, под $\mathscr{L}(X)$ должно пониматься пространство линейных непрерывных операторов на $X$, также наделенное подходящей топологией, а модули и тензорное произведение тоже считаются топологическими в каком-нибудь разумном смысле.)

Понятно, что если для какого-то (бесконечномерного) $X$ такое представление справедливо, то автоматически мы получаем решение следующей проблемы:

ПроБЛЕмА. Можно ли описать топологические модули над топологической алгеброй $\mathscr{L}(X)$ операторов на каком-нибудв (бесконечномерном) топологическом векторном пространстве $X$ ?

В функциональном анализе эта проблема выглядит совершенно безнадежной: даже для самого популярного класса банаховых пространств (и модулей) не найдено никаких примеров, когда подобное описание было бы возможно.

Есть, правда, два близких к этой теме класса задач, изучаемых специалистами. Это, во-первых, описание модулей над (неунитальными) алгебрами $\mathscr{K}(X)$ и $\mathscr{N}(X)$ компактных и ядерных операторов на банаховом пространстве $X$. Успехи в этом направлении скромны и на сегодняшний день ограничиваются гильбертовым случаем: для алгебр $\mathscr{K}(H)$ и $\mathscr{N}(H)$ операторов на гильбертовом пространстве $H$ описаны односторонние замкнутые идеалы и гильбертовы модули [2, гл. 3, теоремы 2.5 и 2.12].

И, во-вторых, это описание замкнутых двусторонних идеалов в банаховой алгебре $\mathscr{B}(X)$ ограниченных операторов на банаховом пространстве $X$. Эта деятельность, начавшись с работы Калкина [3] 1941 г., привела к настоящему времени к описанию решеток замкнутых двусторонних идеалов в $\mathscr{B}(X)$ для нескольких классических пространств $\left(X=c_{0}, \ell_{p}, 1 \leqslant p<\infty\right)$ и одного специального $\left(X=\left(\bigoplus \ell_{2}^{n}\right)_{c_{0}}\right)$ - см. обзор в [4].

В свете этого довольно неожиданным должно оказаться наблюдение, что формулу (1) удается обобщить на случай, когда $X$ принадлежит весьма широкому классу так называемых стереотипных пространств со свойством стереотипной аппроксимации. Это и составляет предмет исследования нашей работы.

Стереотипные пространства изучались автором в работах [5-7], и определяются они следующим образом. Пусть $X$ - локально выпуклое пространство 
над $\mathbb{C}$. Обозначим через $X^{\star}$ пространство линейных непрерывных функционалов $f: X \rightarrow \mathbb{C}$, наделенное топологией равномерной сходимости на вполне ограниченных множествах в $X$. Пространство $X$ называется стереотипнъм, если естественное отображение

$$
i_{X}: X \rightarrow\left(X^{\star}\right)^{\star}, \quad i_{X}(x)(f)=f(x), \quad x \in X, f \in X^{\star},
$$

является изоморфизмом локально выпуклых пространств. Оказывается, что стереотипные пространства образуют весьма широкий класс, включающий в себя, в частности, все пространства Фреше (а значит, и все банаховы пространства).

Отсылая читателя за подробностями к [6], мы в нескольких фразах упомянем здесь те конструкции теории стереотипных пространств, которые понадобятся нам для формулировки главного результата.

Прежде всего, всякое замкнутое подпространство $Y$ в стереотипном пространстве $X$ обладает естественной структурой стереотипного пространства; пространство $Y$ с этой структурой называется непосредственным подпространством в $X$. Несколько огрубляя картину, можно сказать, что факторпространство $X / Y$ стереотипного пространства $X$ по любому замкнутому подпространству $Y$ также обладает структурой стереотипного пространства. Вместе эти две конструкции превращают класс $\mathfrak{S t e ~ c т е р е о т и п н ы х ~ п р о с т р а н с т в ~ в ~ п р е д а б е л е в у ~}$ категорию (с линейными непрерывными отображениями в качестве морфизмOB).

Далее, категория $\mathfrak{S t e ~ о б л а д а е т ~ д в у м я ~ е с т е с т в е н н ы м и ~ т е н з о р н ы м и ~ п р о и з в е д е - ~}$ ниями $\circledast$ и $\odot$, переходящими друг в друга под действием операции $X \mapsto X^{\star}$ :

$$
(X \circledast Y)^{\star}=X^{\star} \odot Y^{\star}, \quad(X \odot Y)^{\star}=X^{\star} \circledast Y^{\star} .
$$

Первое из них, $\circledast$, совпадает с обычным проективным тензорным произведением на классе пространств Фреше и потому называется проективным, а второе, $\odot$, совпадает с инъективным тензорным произведением на классе пространств Фреше со свойством (классической) аппроксимации и потому называется ингективным. Эти два тензорных произведения связаны так называемым преобразованием Гротендика

$$
@_{X, Y}: X \circledast Y \rightarrow X \odot Y,
$$

которое является естественным преобразованием функтора $\circledast$ в функтор $\odot$.

Cтереотипная алгебра $A$ определяется как стереотипное пространство с (ассоциативной) операцией умножения $(a, b) \mapsto a \cdot b$, такой, что для всякого компакта $K \subset A$ и любой окрестности нуля $U \subset A$ найдется такая окрестность нуля $V \subset A$, что $K \cdot V \subseteq U$ и $V \cdot K \subseteq U$. Cтереотипный модуль $M$ над $A$ есть стереотипное пространство с (ассоциативной) операцией умножения $(a, x) \mapsto a \cdot x$ на элементы из $A$, такой, что для любых компактов $K \subset A$ и $L \subset M$ и любой окрестности нуля $U \subset M$ найдутся такие окрестности нуля $V \subset M$ и $W \subset A$, что $K \cdot V \subseteq U$ и $W \cdot L \subseteq U$. Эти определения эквивалентны тому, что операции умножения продолжаются до непрерывных отображений $A \circledast A \rightarrow A$ и $A \circledast M \rightarrow M$.

Для всякого стереотипного пространства $X$ алгебра $\mathscr{L}(X)$ линейных непрерывных операторов на $X$ обладает естественной топологией, превращающей 
$\mathscr{L}(X)$ в стереотипную алгебру. Эта топология представляет собой некое усиление топологии равномерной сходимости на компактах, а точнее - результат применения к этой топологии так называемой операции псевдонасыщения (см. [6]).

Говорят, что стереотипное пространство $X$ обладает свойством cmepeomunной аппроксимации, если тождественный оператор в $\mathscr{L}(X)$ приближается конечномерными (в топологии пространства $\mathscr{L}(X))$. Это равносильно тому, что для любого стереотипного пространства $Y$ преобразование Гротендика

$$
@_{X, Y}: X \circledast Y \rightarrow X \odot Y
$$

является биморфизмом стереотипных пространств (т. е. инъективным отображением с плотным образом). Пространств со свойством стереотипной аппроксимации довольно много, потому что это свойство лежит между классическим свойством аппроксимации и существованием базиса в $X$.

Если зафиксировать $X$, то для всякого другого стереотипного пространства $E$ тензорные произведения $E \circledast X$ и $E \odot X$ будут стереотипными модулями над стереотипной алгеброй $\mathscr{L}(X)$. Эти два модуля связаны между собой преобразованием Гротендика

$$
@_{E, X}: E \circledast X \rightarrow E \odot X .
$$

Если пространство $X$ обладает свойством стереотипной аппроксимации, то это отображение будет биморфизмом (т.е. плотным вложением). Поэтому связь между $E \circledast X$ и $E \odot X$ удобно представлять себе как вложение с плотным образом

$$
E \circledast X \subseteq E \odot X
$$

в котором $E \circledast X$ и $E \odot X$ можно в первом приближении мыслить как специального рода пополнения алгебраического тензорного произведения $E \otimes X$ относительно двух разных топологий (одна из которых слабее другой).

Основной результат настоящей работы состоит в том, что любой стереотипный $\mathscr{L}(X)$-модуль $M$ лежит между $E \circledast X$ и $E \odot X$ для некоторого однозначно определенного стереотипного пространства $E$ :

$$
E \circledast X \subseteq M \subseteq E \odot X .
$$

Его более точная формулировка выглядит так:

TEOPEмA 1.1. Пусть $X$ - стереотипное пространство со свойством стереотипной аппроксимации. Тогда для всякого левого стереотипного модуля $M$ над $\mathscr{L}(X)$ найдутся единственное ( с точностью до изоморфизма) стереотипное пространство $\mathrm{E}(M)$ и два биморфизма левых стереотипных $\mathscr{L}(X)$-модулей $(m . е$. два $\mathscr{L}(X)$-линейных непрерывных отображения с нулевым ядром $и$ плотным образом) $\rho_{M}: \mathrm{E}(M) \circledast X \rightarrow M u \sigma_{M}: M \rightarrow \mathrm{E}(M) \odot X$, такие, что следующая диаграмма коммутативна:

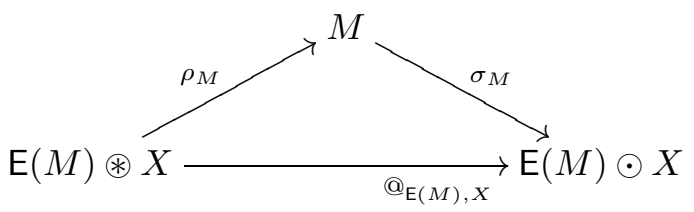

При этом

(а) $\mathrm{E}(M)$ является непосредственным подпространством в $M$; 
(b) если $\mu: M \rightarrow N-$ морфизм стереотипных модулей над $\mathscr{L}(X)$, то однозначно определен морфизм стереотипных пространств $\mathrm{E}(\mu): \mathrm{E}(M) \rightarrow \mathrm{E}(N)$, такой, что коммутативна диаграмма

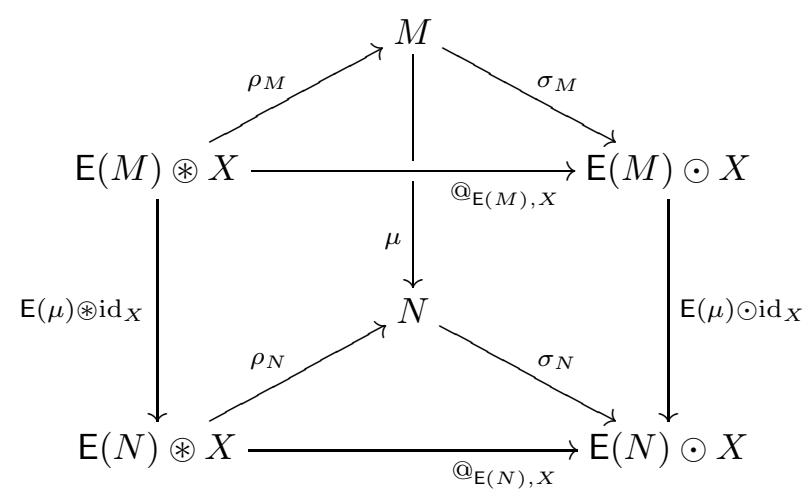

(с) отображение $\mathrm{E}(\cdot)$ является ковариантным функтором из категории $\mathscr{L}(X)$ Ste левых стереотипных модулей над $\mathscr{L}(X)$ в категорию Ste стереотипных пространств над $\mathbb{C}$, а семейства морфизмов $\left\{\rho_{M} ; M \in \mathscr{L}(X) \mathfrak{S t e}\right\} u$ $\left\{\sigma_{M} ; M \in \mathscr{L}(X) \mathfrak{S t e}\right\}$ являются естественными преобразованиями функтора $\mathrm{E}(\cdot) \circledast X$ в тождественный бунктор на категории $\mathscr{L}(X)$ Ste $и$ тождественного функтора в функтор $\mathrm{E}(\cdot) \odot X$ соответственно.

Доказательство этого факта мы приведем ниже, а сейчас отметим два важных следствия из него.

СлЕДСтвиЕ 1.1. Пусть $X$ - ядерное пространство Фреше с базисом ${ }^{1)}$. Тогда для всякого левого модуля Фреше $M$ над стереотипной алгеброй операторов $\mathscr{L}(X)$ существует единственное (с точностью до изоморфизма) пространство Фреше $E$, такое, что $M$ изоморфен проективному тензорному произведению пространств Фреше $E$ и $X$,

$$
M \cong E \widehat{\otimes} X
$$

ДокАЗАТЕЛьство. Положим $E=\mathrm{E}(M)$. Пункт (а) теоремы 1.1 означает в случае метризуемого модуля $M$, что $E$ есть замкнутое подпространство в $M$, а значит, должно быть пространством Фреше. Отсюда в силу [6, Theorem 7.17 and 7.21] получаем цепочку изоморфизмов

$$
\begin{gathered}
X \text { ядерное } \\
\stackrel{\downarrow}{\unrhd} E \cong E \check{\otimes} X \cong E \odot X,
\end{gathered}
$$

1) Существование базиса в $X$ здесь постулируется для того, чтобы $X$ заведомо обладало свойством стереотипной аппроксимации (и, как следствие, чтобы к $X$ можно было применять теорему 1.1). Дело в том, что к настоящему времени неизвестно, совпадает ли свойство стереотипной аппроксимации с обычным свойством классической аппроксимации. Ясно только что из существования базиса следует свойство стереотипной аппроксимации, а из него следует свойство классической аппроксимации. Поэтому, если мы хотим, чтобы к $X$ применялась теорема 1.1, нам приходится требовать существования базиса, чтобы сформулировать результат в общепринятых терминах. Поскольку это единственная причина, почему нам здесь нужно это условие, очень возможно, что его можно отбросить (даже если обнаружится, что стереотипная аппроксимация в действительности не совпадает с классической). Но автору такая редукция неизвестна. 
означающую, что преобразование Гротендика @ $\mathrm{E}(M), X: \mathrm{E}(M) \circledast X \rightarrow \mathrm{E}(M) \odot X$ является изоморфизмом пространств Фреше. Теперь из равенства $\sigma_{M} \circ \rho_{M}=$ $@_{\mathrm{E}(M), X}$ и из того факта, что $\sigma_{M}$ и $\rho_{M}-$ биморфизмы, следует, что $\sigma_{M}$ и $\rho_{M}-$ изоморфизмы.

СлЕдствиЕ 1.2 (характеризация простых модулей над $\mathscr{L}(X))$. Ecли $X-$ стереотипное пространство со свойством стереотипной аппроксимации, то для стереотипного левого модуля $M \neq 0$ над $\mathscr{L}(X)$ следующие условия эквивалентны:

(i) любой мономорбизм $\mu: N \rightarrow M$ стереотипных левых $\mathscr{L}(X)$-модулей является либо нулевым, либо изоморфизмом;

(ii) любой эпиморфизм $\mu: M \rightarrow N$ стереотипных левых $\mathscr{L}(X)$-модулей является либо нулевым, либо изоморфизмом;

(iii) $M \cong X$.

\section{Для доказательства нам понадобится следующая}

ЛЕмма 1.1. Если $X$ - локально выпуклое пространство и $a, b \in X-\partial в а$ ненулевых вектора, то найдется автоморфизм (т.е. биективное линейное отображение, непрерьвное в обе сторонъ) $\varphi: X \rightarrow X$, переводящий $а$ в $b$ :

$$
\varphi(a)=b
$$

ДокАЗАТЕЛьСтво. Если $b=\lambda a$, то можно положить $\varphi(x)=\lambda x$. Поэтому важно рассмотреть случай, когда $a$ и $b$ линейно независимы. Рассмотрим линейную оболочку $P=\operatorname{span}\{a, b\}$. Пусть $Q-$ замкнутое подпространство в $X$, дополняющее $P, X=P \oplus Q$. Тогда оператор $\varphi$ можно определить правилами $\left.\varphi\right|_{Q}=\operatorname{id}_{Q}, \varphi(a)=b, \varphi(b)=a$.

ДокАЗАТЕЛЬСтво СЛЕДстВия 1.2. 1. Докажем сначала импликации (iii) $\Rightarrow(\mathrm{i})$ и $(\mathrm{iii}) \Rightarrow(\mathrm{ii})$.

Начнем с (iii) $\Rightarrow$ (i). Пусть $\mu: N \rightarrow X$ - ненулевой мономорфизм. Зафиксируем $n \in N$, такое, что $\mu(n)=a \neq 0$. Тогда (по лемме 1.1) векторы

$$
x=\varphi(a)=\mu(\varphi \cdot n), \quad \varphi \in \mathscr{L}(X),
$$

пробегают все пространство $X$. Значит, $\mu: N \rightarrow X-$ сюръекция и, следовательно, биекция. Нам остается проверить, что обратное отображение $\mu^{-1}: X \rightarrow N$ непрерывно. Пусть $x_{i}$ - направленность в $X$, стремящаяся к нулю,

$$
x_{i} \stackrel{X}{\longrightarrow} 0, \quad i \rightarrow \infty .
$$

Покажем, что тогда направленность $\mu^{-1}\left(x_{i}\right)$ стремится к нулю в $N$. Зафиксируем для этого функционал $g \in X^{\star}$, такой, что $g(a)=1$, и заметим, что

$$
g \odot x_{i} \cdot n=g \odot x_{i} \cdot \mu^{-1}(a)=\mu^{-1}\left(g \odot x_{i} \cdot a\right)=\mu^{-1}\left(g(a) \cdot x_{i}\right)=\mu^{-1}\left(x_{i}\right) .
$$

Теперь получаем

$$
x_{i} \stackrel{X}{\longrightarrow} 0 \Longrightarrow g \odot x_{i} \stackrel{\mathscr{L}(X)}{\longrightarrow} 0 \Longrightarrow \mu^{-1}\left(x_{i}\right)=g \odot x_{i} \cdot n \stackrel{N}{\longrightarrow} 0 .
$$

После того как импликация (iii) $\Rightarrow$ (i) доказана, импликация (iii) $\Rightarrow$ (ii) получается переходом к сопряженным модулям. Чтобы это понять, нужно заметить, что алгеброй с противоположным умножением для $\mathscr{L}(X)$ будет алгебра $\mathscr{L}\left(X^{\star}\right)$ операторов на сопряженном пространстве,

$$
\mathscr{L}(X)^{\mathrm{op}} \cong \mathscr{L}\left(X^{\star}\right)
$$


(изоморфизм определяется переходом к сопряженному отображению $\varphi \mapsto \varphi^{\star}$ ). Поэтому если дан ненулевой эпиморфизм $\mu: X \rightarrow N$ левы $\mathscr{L}(X)$-модулей, то сопряженное отображение $\mu^{\star}: N^{\star} \rightarrow X^{\star}$ будет ненулевым мономорфизмом $n p a в ы x \mathscr{L}(X)$-модулей, или, что то же самое, ненулевым мономорфизмом левы модулей над $\mathscr{L}(X)^{\text {op }}=\mathscr{L}\left(X^{\star}\right)$. Про такие мономорфизмы мы уже доказали, что они являются изоморфизмами. Значит, $\mu^{\star}: N^{\star} \rightarrow X^{\star}-$ изоморфизм и, следовательно, $\mu: X \rightarrow N$ - тоже изоморфизм.

2. Теперь убедимся, что, обратно, справедливы импликации (i) $\Rightarrow$ (iii) и (ii) $\Rightarrow$ (iii). Здесь нужно лишь проверить, что в случаях (i) и (ii) имеет место равенство $\mathrm{E}(M)=\mathbb{C}$. Действительно, если это не так, т. е. $\mathrm{E}(M) \neq \mathbb{C}$, то можно взять вложение $\eta: \mathbb{C} \rightarrow \mathrm{E}(M)$, не являющееся изоморфизмом, и тогда композиция

$$
X=\mathbb{C} \circledast X \stackrel{\eta \circledast \mathrm{id}_{X}}{\longrightarrow} \mathrm{E}(M) \circledast X \stackrel{\rho_{M}}{\longrightarrow} M,
$$

не будучи нулевой, не является изоморфизмом. Значит, условие (i) не может выполняться.

Точно так же при $\mathrm{E}(M) \neq \mathbb{C}$ можно взять ненулевую проекцию $\theta: \mathrm{E}(M) \rightarrow \mathbb{C}$, не являющуюся изоморфизмом, и тогда композиция

$$
M \stackrel{\sigma_{M}}{\longrightarrow} \mathrm{E}(M) \odot X \stackrel{\theta \odot \text { id }_{X}}{\longrightarrow} \mathbb{C} \odot X=X
$$

не будет нулевой (потому что $\sigma_{M}$ - эпиморфизм, а значит, из равенства $\theta \odot$ $\operatorname{id}_{X} \circ \sigma_{M}=0$ должно следовать $\theta \odot \operatorname{id}_{X}=0$, т. е. $\left.\theta=0\right)$ и не является изоморфизмом (потому что для ненулевых $x \in X$ и $e \in \operatorname{Ker}(\theta)$ мы получаем $e \circledast x \neq 0$ и, значит, $\rho_{M}(e \circledast x) \neq 0$, но, с другой стороны, $\left(\theta \odot \operatorname{id}_{X}\right)\left[\sigma_{M}\left(\rho_{M}(e \circledast x)\right)\right]=$ $\theta(e) \odot x=0)$. Следовательно, условие (ii) не может выполняться.

\section{§2. Доказательство теоремы 1.1}

Мы всюду используем терминологию и обозначения работы [6].

Лемма 2.1. Пусть $X-$ стереотипное пространство и $M \in \mathscr{L}(X)$ Ste. Тогда $\forall g \neq 0 \in X^{\star} \quad \forall a \in X \quad \forall f \in X^{\star} \quad \forall m \in M \quad \exists n \in M \quad f \odot a \cdot m=g \odot a \cdot n$.

ДокАЗАтЕЛЬСтво. Если $f=0$, то можно положить $n=0$. Поэтому мы будем считать, что $f \neq 0$. Тогда по лемме 1.1 найдется автоморфизм $\varphi^{\star}: X^{\star} \rightarrow X^{\star}$, такой, что

$$
\varphi^{\star}(f)=g .
$$

Ему соответствует автоморфизм $\varphi: X \rightarrow X$. Положив $n=\varphi^{-1} \cdot m$, мы получим

$$
f \odot a \cdot m=f \odot a \cdot \varphi \cdot \varphi^{-1} \cdot m=\underbrace{(f \circ \varphi)}_{\substack{\varphi^{\star}(f) \\ \|}} \odot a \cdot \underbrace{\varphi^{-1} m}_{\substack{\| \\ n}}=g \odot a \cdot n .
$$

Лемма 2.2. Пусть $X$ и $E$ - произволъные стереотипнъе пространства,

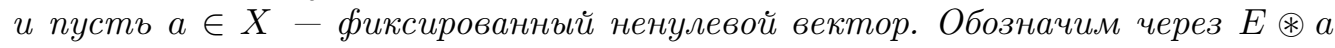
$u E \odot$ а непосредственные подпространства в $E \circledast X u E \odot X$, образованные векторами вида $е \circledast а$ и $е \odot а(e \in E)$ соответственно. Рассмотрим отображения

$$
\zeta: E \rightarrow E \circledast a=\{e \circledast a ; e \in E\}, \quad \zeta(e)=e \circledast a,
$$


$u$

$$
\eta: E \rightarrow E \odot a=\{e \odot a ; e \in E\}, \quad \eta(e)=e \odot a .
$$

Пусть, кроме того, ๔ обозначает ограничение преобразования Гротендика @ : $E \circledast X \rightarrow E \odot X$ на подпространство $E \circledast a:$

$$
\widetilde{@}=\left.@\right|_{E \circledast a}
$$

Тогда в (коммутативной) диаграмме

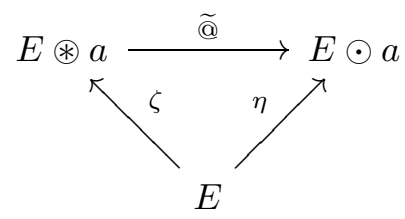

все отображсеня являются изоморфизмами.

ДокАЗАтельство. Очевидно, достаточно доказать, что $\zeta$ и $\eta$ являются изоморфизмами. Для этого нужно рассмотреть их как отображения со значениями в пространствах $E \circledast X$ и $E \odot X$, т. е. как отображения $\zeta: E \rightarrow E \circledast X$ и $\eta: E \rightarrow E \odot X$, и заметить, что тогда они будут коретракциями. Действительно, если зафиксировать функционал $g \in X^{\star}$, такой, что $g(a)=1$, то, положив

$$
\pi: E \circledast X \rightarrow E, \quad \pi(e \circledast x)=g(x) e, \quad e \in E,
$$

и

$$
\tau: E \odot X=E \oslash X^{\star} \rightarrow E, \quad \tau(\varphi)=\varphi(g), \quad \varphi \in E \oslash X^{\star},
$$

мы получим, во-первых, что

$$
\forall e \in E \quad \pi(\zeta(e))=\pi(e \circledast a)=g(a) \cdot e=e,
$$

т. е. $\pi \circ \zeta=\operatorname{id}_{E}$, и, во-вторых, что

$$
\forall e \in E \quad \tau(\eta(e))=\tau(e \odot a)=(e \odot a)(g)=g(a) \cdot e=e,
$$

т. е. $\tau \circ \eta=\mathrm{id}_{E}$.

ДОКАЗАТЕЛЬСТВо тЕОРЕМЫ 1.1. 1. Зафиксируем с начала и до конца доказательства элемент $a \in X$ и функционал $g \in X^{\star}$, такие, что

$$
g(a)=1 .
$$

2. Заметим, что оператор $g \odot a$ является проектором:

$$
g \odot a \cdot g \odot a=\underbrace{g(a)}_{1} \cdot g \odot a=g \odot a .
$$

Поэтому отображение

$$
\pi: M \rightarrow M, \quad \pi(n)=g \odot a \cdot n,
$$

тоже является проектором: $\pi^{2}=\pi$.

3. Положим

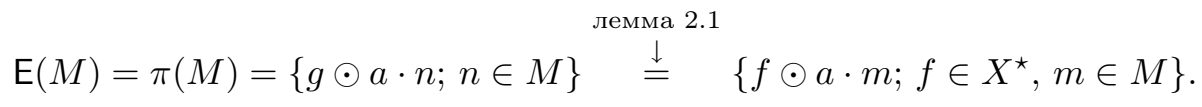


Поскольку $\pi$ - проектор, $\mathrm{E}(M)$ является замкнутым подпространством в $M$. Мы наделяем его топологией непосредственного подпространства в $M$. Заметим, кроме того, что, поскольку проектор $\pi: M \rightarrow \mathrm{E}(M)$ оставляет на месте элементы своего образа $\mathrm{E}(M)$, выполняется тождество

$$
g \odot a \cdot e=\pi(e)=e, \quad e \in \mathrm{E}(M) .
$$

4. В силу последнего равенства в цепочке (4) для любых $f \in X^{\star}$ и $m \in M$ имеем $f \odot a \cdot m \in \mathrm{E}(M)$. Поэтому определено отображение

$$
\sigma_{M}: M \rightarrow \mathrm{E}(M) \odot X=\mathrm{E}(M) \oslash X^{\star}, \quad \sigma_{M}(m)(f)=f \odot a \cdot m .
$$

Заметим, что

1) $\sigma_{M}$ является морфизмом $\mathscr{L}(X)$-модулей, так как при $\alpha \in \mathscr{L}(X), m \in M$, $f \in X^{\star}$ получаем

$$
\sigma_{M}(\alpha m)(f)=f \odot a \cdot \alpha m=(f \circ \alpha) \odot a \cdot m=\sigma_{M}(m)(f \circ \alpha)=\left\{\alpha\left[\sigma_{M}(m)\right]\right\}(f),
$$

откуда следует, что $\sigma_{M}(\alpha m)=\alpha\left[\sigma_{M}(m)\right]$;

2) $\sigma_{M}$ инъективно, поскольку равенство $\sigma_{M}(m)=0$ рождает следующую цепочку импликаций: $f \odot a \cdot m=0$ для любого $f \in X^{\star} \Longrightarrow f \odot \alpha(a) \cdot m=$ $\alpha \cdot f \odot a \cdot m=0$ для любых $f \in X^{\star}$ и $\alpha \in \mathscr{L}(X) \Longrightarrow f \odot x \cdot m=0$ для любых $f \in X^{\star}$ и $x \in X \Longrightarrow \beta \cdot m=0$ для произвольного конечномерного оператора $\beta \in \mathscr{L}(X) \Longrightarrow 1 \cdot m=m=0$ (поскольку конечномерные операторы аппроксимируют единичный в $\mathscr{L}(X))$.

5. Определим отображение $\rho_{M}$ формулой

$$
\rho_{M}: \mathrm{E}(M) \circledast X \rightarrow M, \quad \rho_{M}(e \circledast x)=g \odot x \cdot e, \quad x \in X, e \in \mathrm{E}(M) .
$$

Из непрерывности билинейных форм $(e, x) \mapsto e \circledast x,(f, x) \mapsto f \odot x$ и $(\alpha, m) \mapsto \alpha \cdot m$ следует, что билинейная форма $(e, x) \mapsto g \odot x \cdot e$ тоже непрерывна (в смысле определения [6]). Поэтому в силу универсальности тензорного произведения $\circledast$ (см. [6, Theorem 7.3]) формула (6) однозначно определяет непрерывное отображение $\rho_{M}: \mathrm{E}(M) \circledast X \rightarrow M$.

Заметим, что

1) $\rho_{M}$ является морфизмом $\mathscr{L}(X)$-модулей; это следует из цепочки равенств $\rho_{M}(\alpha \cdot e \circledast x)=\rho_{M}(e \circledast \alpha(x))=g \odot \alpha(x) \cdot e=\alpha \cdot g \odot x \cdot e=\alpha \cdot \rho_{M}(e \circledast x), \quad \alpha \in \mathscr{L}(X) ;$

2) $\rho_{M}$ - эпиморфизм; чтобы это понять, зафиксируем $m \in M$ и заметим, что, поскольку для всякого $f \in X^{\star}$ вектор $e=f \odot a \cdot m$ лежит в $\mathrm{E}(M)$, справедливо тождество

$$
\rho_{M}((\underbrace{f \odot a \cdot m}_{e}) \circledast x)=g \odot x \cdot \underbrace{f \odot a \cdot m}_{e}=f \odot x \cdot m,
$$

из которого следует, что элементы вида $f \odot x \cdot m$ принадлежат образу отображения $\rho_{M}$; это, в свою очередь, означает, что элементы вида $\beta \cdot m$, где $\beta-$ конечномерный оператор, тоже лежат в $\operatorname{Im} \rho_{M}$; поскольку конечномерные операторы аппроксимируют единичный в $\mathscr{L}(X)$, элемент $1 \cdot m=m$ тоже принадлежит $\operatorname{Im} \rho_{M}$ (по определению считается, что образ $\operatorname{Im} \mu$ морфизма $\mu: X \rightarrow Y$ есть замыкание элементов вида $\mu(x), x \in X)$; это верно для любого $m \in M$, а значит, $\rho_{M}$ - действительно эпиморфизм.

6. Докажем теперь коммутативность диаграммы (2), т. е. справедливость равенства

$$
\sigma_{M} \circ \rho_{M}=@_{\mathrm{E}(M), X} .
$$


Для этого нужно зафиксировать $x \in X$ и $e \in \mathrm{E}(M)$ и проверить, что

$$
\sigma_{M}\left(\rho_{M}(e \circledast x)\right)=e \odot x .
$$

Действительно, для любого $f \in X^{\star}$ получаем

$$
\begin{aligned}
& \sigma_{M}(\underbrace{\left(\rho_{M}(e \circledast x)\right.}_{\begin{array}{c}
g \odot x \cdot e \\
\text { в силу (6) }
\end{array}})(f)=\sigma_{M}(g \odot x \cdot e)(f)=\underbrace{f \odot a \cdot g \odot x}_{\begin{array}{c}
\| \\
f(x) \cdot g \odot a \\
\text { в силу }[6,(9.7)]
\end{array}} \cdot e=f(x) \cdot \underbrace{g \odot a \cdot e}_{\begin{array}{c}
\| \\
e \\
\text { в силу (5) }
\end{array}} \\
& =f(x) \cdot e=[e \odot x](f)
\end{aligned}
$$

7. Покажем, что $\rho_{M}$ и $\sigma_{M}-$ биморфизмы. Это следует из диаграммы (2): поскольку $X$ обладает свойством стереотипной аппроксимации, преобразование Гротендика @ $@_{\mathrm{E}(M), X}$ должно быть биморфизмом. Значит, во-первых,

$$
\mathrm{E}(M) \odot X=\operatorname{Im} @_{\mathrm{E}(M), X} \subseteq \operatorname{Im} \sigma_{M}
$$

и поэтому $\sigma_{M}$ - эпиморфизм, а поскольку, как мы уже показали, $\sigma_{M}-$ мономорфизм, мы получаем, что $\sigma_{M}$ - биморфизм.

И, во-вторых,

$$
\text { Ker } \rho_{M} \subseteq \operatorname{Ker} @_{\mathrm{E}(M), X}=0,
$$

а значит, $\rho_{M}$ - мономорфизм; кроме того, как мы знаем, $\rho_{M}$ - эпиморфизм. Поэтому $\rho_{M}$ должен быть биморфизмом.

8. Покажем теперь, что если для модуля $M$ имеется коммутативная диаграммa

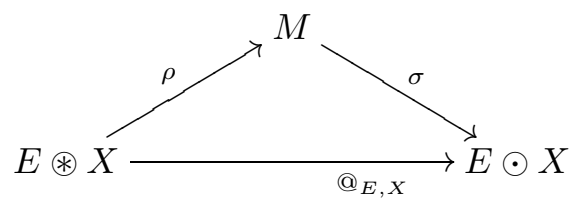

где $\rho$ и $\sigma$ - биморфизмы, то пространство $E$ автоматически изоморфно $\mathrm{E}(M)$.

Это можно сделать, достроив диаграмму (7) до коммутативной диаграммы

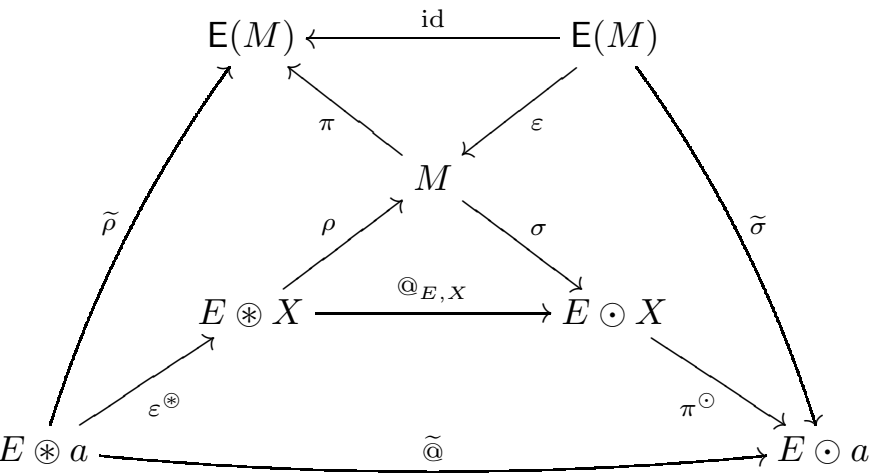

в которой $\varepsilon$ и $\varepsilon^{\circledast}-$ естественные вложения, $\pi$ и $\pi^{\odot}$ - отображения умножения на одномерный оператор $g \odot a$, а $\widetilde{\rho}, \widetilde{\sigma}$ и @ определяются формулами

$$
\widetilde{\rho}=\pi \circ \rho \circ \varepsilon^{\circledast}, \quad \widetilde{\sigma}=\pi^{\odot} \circ \sigma \circ \varepsilon, \quad \widetilde{@}=\pi^{\odot} \circ @_{E, X} \circ \varepsilon^{\circledast} .
$$

Отметим здесь два факта: 
(i) @ есть ограничение отображения @ $E, X$ на подпространство $E \circledast a$,

$$
\widetilde{@}(e \circledast a)=e \odot a, \quad e \in E,
$$

и поэтому по лемме 2.2 является изоморфизмом;

(ii) $\widetilde{\sigma}$ есть мономорфизм.

Действительно, во-первых, для всякого $e \in E$ получаем

$$
e \circledast a \stackrel{\varepsilon^{\circledast}}{\longmapsto} e \circledast a \stackrel{\varrho_{E, X}}{\longmapsto} e \odot a \stackrel{\pi^{\odot}}{\longmapsto}(g \odot a) \cdot(e \odot a)=g(a) \cdot(e \odot a)=e \odot a .
$$

Во-вторых, если $\widetilde{\sigma}(e)=0$ для какого-нибудь элемента $e \in \mathrm{E}(M)$, то

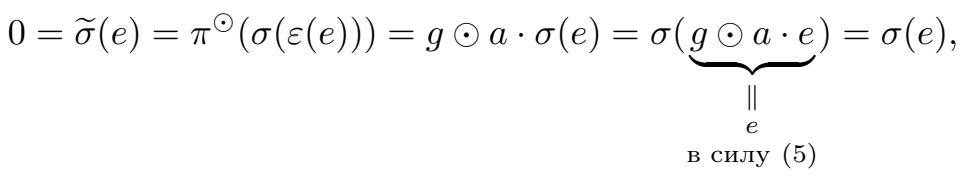

а поскольку $\sigma$ - мономорфизм, получаем, что $e=0$.

Выбросив теперь из диаграммы (8) внутренние стрелки, мы придем к коммутативной диаграмме

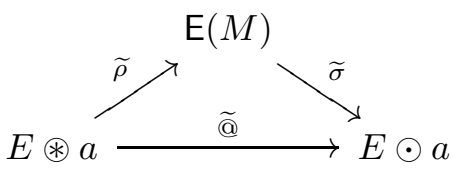

в которой $\widetilde{@}-$ изоморфизм (в силу (i)). Значит, $\widetilde{\sigma}$ - ретракция. В то же время (в силу (ii)) $\widetilde{\sigma}$ является мономорфизмом. Следовательно, $\widetilde{\sigma}-$ изоморфизм и вообще все стрелки в (9) являются изоморфизмами. Таким образом, снова применяя лемму 2.2 , получаем цепочку изоморфизмов

$$
E \cong E \circledast a \cong E \odot a \cong \mathrm{E}(M) .
$$

9. Нам остается показать, что если $\mu: M \rightarrow N-$ морфизм стереотипных $\mathscr{L}(X)$-модулей, то определен морфизм $\mathrm{E}(\mu): \mathrm{E}(M) \rightarrow \mathrm{E}(N)$, такой, что коммутативна диаграмма (3). Для этого нужно заметить, что $\mathrm{E}(M)$ и $\mathrm{E}(N)$ определены формулой (4),

$$
\mathrm{E}(M)=\{g \odot a \cdot m ; m \in M\}, \quad \mathrm{E}(N)=\{g \odot a \cdot n ; n \in N\},
$$

и, поскольку отображение $\mu: M \rightarrow N$ коммутирует со всеми операторами $\alpha \in$ $\mathscr{L}(X)$, в частности с $\alpha=g \odot a$, оно должно переводить $\mathrm{E}(M)$ в $\mathrm{E}(N)$. Поэтому мы можем положить $\mathrm{E}(\mu)=\left.\mu\right|_{\mathrm{E}(M)}$.

Проверим коммутативность диаграммы (3). Коммутативность правого параллелограмма, т. е. равенство

$$
\left(\mathrm{E}(\mu) \odot \operatorname{id}_{X}\right) \circ \sigma_{M}=\sigma_{N} \circ \mu,
$$

проверяется прямым вычислением: для $m \in M$ и $f \in X^{\star}$ имеем

$$
\begin{gathered}
{\left[\left(\mathrm{E}(\mu) \odot \mathrm{id}_{X}\right) \circ \sigma_{M}\right](m)(f)=\left(\mathrm{E}(\mu) \odot \mathrm{id}_{X}\right)\left[\sigma_{M}(m)\right](f)=\left(\mathrm{E}(\mu) \oslash \mathrm{id}_{X}^{\star}\right)\left[\sigma_{M}(m)\right](f)} \\
=\left(\mathrm{E}(\mu) \circ \sigma_{M}(m) \circ \mathrm{id}_{X}^{\star}\right)(f)=\mathrm{E}(\mu)\left[\sigma_{M}(m)(f)\right]=\mu[f \odot a \cdot m] \\
=f \odot a \cdot \mu[m]=\sigma_{N}(\mu(m))(f)=\left[\sigma_{N} \circ \mu\right](m)(f) .
\end{gathered}
$$


Затем таким же прямым вычислением проверяется коммутативность левого параллелограмма, т. е. равенство

$$
\mu \circ \rho_{M}=\rho_{N} \circ\left(\mathrm{E}(\mu) \circledast \mathrm{id}_{X}\right) ;
$$

таким образом, при $e \in \mathrm{E}(M)$ и $x \in X$ получаем

$$
\mu\left(\rho_{M}(e \circledast x)\right)=\mu(g \odot x \cdot e)=g \odot x \cdot \mu(e)=\rho_{N}(\mu(e) \circledast x)=\rho_{N}(\mathrm{E}(\mu)(e) \circledast x) .
$$

И наконец, коммутативность прямоугольника очевидна:

$$
\left(\mathrm{E}(\mu) \odot \operatorname{id}_{X}\right) \circ @_{\mathrm{E}(M), X}=@_{\mathrm{E}(N), X} \circ\left(\mathrm{E}(\mu) \circledast \operatorname{id}_{X}\right) .
$$

Автор выражает признательность рецензентам за ряд весьма полезных замечаний, способствовавших улучшению статьи.

\section{ЛитеРАТУРА}

1. Пирс Р. Ассоциативные алгебры. Мир, М., 1986.

2. Хелемский А. Я. Банаховы и полинормированные алгебры: общая теория, представления, гомологии. Наука, М., 1989.

3. Calkin J. W. Two-sided ideals and congruences in the ring of bounded operators in Hilbert space. Ann. of Math., 42, 839-873 (1941).

4. Laustsen N. J., Loy R. J., Read C. J. The lattice of closed ideals in the Banach algebra of operators on certain Banach spaces. J. Funct. Anal., 214, 106-131 (2004).

5. Akbarov S. S. Stereotype spaces, algebras, homologies: An outline. In: Topological Homology (A. Ya. Helemskii, ed.), Nova Science Publishers, 2000, pp. 1-29.

6. Akbarov S. S. Pontryagin duality in the theory of topological vector spaces and in topological algebra. J. Math. Sci., 113, No. 2, 179-349 (2003).

7. Akbarov S. S. Pontryagin duality and topological algebras. In: Topological Algebras, Their Applications, and Related Topics, Banach Center Publications series, Vol. 67, 2005, pp. 55-71. 